\title{
Co-targeting of Cyclooxygenase-2 and FoxM1 is a viable strategy in inducing anticancer effects in colorectal cancer cells
}

Maqbool Ahmed ${ }^{1 \dagger}$, Azhar R Hussain ${ }^{1 \dagger}$, Abdul K. Siraj ${ }^{1}$, Shahab Uddin ${ }^{1}$, Nasser Al-Sanea ${ }^{2}$, Fouad Al-Dayel ${ }^{3}$, Mohammed Al-Assiri', Shaham Beg ${ }^{1}$ and Khawla S. Al-Kuraya ${ }^{1,5^{*}}$

\begin{abstract}
Background: Cross-talk between deregulated signaling pathways in cancer cells causes uncontrolled growth and proliferation. These cancers cells become more aggressive and quickly develop resistance to therapy. Therefore targeting of these deregulated pathways simultaneously can result in efficient cell death of cancer cells. In this study we investigated co-expression of Cox-2 and FoxM1 in a cohort of colorectal carcinoma (CRC) samples and also examined whether inhibition of Cox-2 and FoxM1 simultaneously can lead to inhibition of cell viability and induction of apoptosis in colorectal cancer cell lines and in vivo xenografts.
\end{abstract}

Methods: Protein expression of Cox-2 and FoxM1 was determined in a large cohort of 770 clinical CRC samples in a tissue micro-array format by immunohistochemistry. Cell death was measured using live dead assay. Apoptosis was measured by annexin V/PI dual staining. Immunoblotting was performed to examine the expression of proteins. Calcusyn software was utilized to estimate the synergistic doses using chou and Talalay method.

Results: Co-expression of Cox-2 and FoxM1 was detected in $33.3 \%$ (232/697) of CRC's and associated with an aggressive phenotype characterized by younger age $(p=0.0191)$, high proliferative index marker; Ki-67 $(p=0.004)$ and MMP-9 $(p=0.0116)$ as well as activation of AKT $(p=0.0214)$. In vitro, inhibition of FoxM1 and Cox-2 with pharmacological inhibitors; Thiostrepton and NS398 resulted in efficient down-regulation of FoxM1 and Cox-2 expression along with in-activation of AKT and inhibition of colony formation, invasion and migratory capability of CRC cells. In addition, there was also inhibition of cell viability and induction of apoptosis via the mitochondrial apoptotic pathway in CRC cell lines. Finally, treatment of CRC xenograft tumors in nude mice with combination of Cox-2 and FoxM1 inhibitors inhibited tumor growth significantly via down-regulation of Cox-2 and FoxM1 expression.

Conclusions: These findings demonstrate that co-expression of Cox-2 and FoxM1 might play a critical role in the pathogenesis of CRC. Therefore, targeting of these pathways simultaneously with sub toxic doses of pharmacological inhibitors can be a potential therapeutic approach for the treatment of this subset of CRC.

Keywords: Colorectal cancer, Cox-2, FoxM1, Cell viability, Colony formation, Invasion

\footnotetext{
* Correspondence: kkuraya@kfshrc.edu.sa

${ }^{\dagger}$ Equal contributors

${ }^{1}$ Human Cancer Genomic Research, Research Center, Riyadh, Saudi Arabia

${ }^{5}$ Al-Faisal University, Riyadh, Saudi Arabia

Full list of author information is available at the end of the article
} 


\section{Background}

Despite increased awareness in the general population regarding colorectal cancer (CRC), it still remains a major cause of mortality and morbidity worldwide [1]. This increase has been attributed to a combination of environmental and genetic factors in the general population [2, 3]. Even though CRC is very well studied along with established diagnostic markers, most of CRC cancers present at late stages of disease and therefore have a poor prognosis [4]. In addition, recurrence and metastasis of CRC also carry a very high mortality rate [5]. Therefore, there is a need for improvement in the diagnosis of CRC as well as identification of newer therapeutic targets that can be specifically drugged to improve the management of these cancers.

An important key survival molecule that is currently being investigated as a molecular marker and a potential therapeutic target is cyclooxygenase-2 (Cox-2) in various cancers. The main function of Cox is to synthesize prostaglandins from arachidonic acid [6]. There are two isoforms of Cox; Cox-1 that is found to be expressed in normal cells [7] and Cox-2 that is preferentially expressed in cancer cells [7] and its expression is enhanced by proinflammatory cytokines and carcinogens $[8,9]$. Cox-2 has been found to be over-expressed by us and others in a variety of cancer including breast, ovary, colorectal, thyroid and lung [10-14]. Prophylactic use of Cox-2 inhibitors such as aspirin has been shown to decrease the incidence of certain cancers [15-18].

Forkhead box protein M1 (FoxM1) is a member of FoxM family that consists of more than 50 proteins that are characterized by a conserved 100 amino acid DNA binding domain $[19,20]$. FoxM1 has also been known to regulate the transcriptional activity of number of genes including cyclin B, cyclin A and Aurora B kinase, which are very important for cell cycle progression and mitotic entry [21-23]. Loss of FoxM1 expression has also been reported to generate mitotic spindle defects leading to mitotic catastrophe [21, 24, 25]. FoxM1 signaling has been implicated to be associated with carcinogenesis of tumor development in CRC as well as other solid tumors [22, 26-33].

A number of dysregulated survival pathways have the ability to cross-talk with each other to increase aggressiveness of various cancers [34, 35]. These cross-talks allow the cancer cell to avoid different in vivo and in vitro threats thereby allowing un-supervised growth and proliferation and the cancers cells become more aggressive and quickly develop resistance to therapy [35]. Inhibiting one pathway may not be enough to elicit a complete response because of the cross-talk with other pathways thereby eliciting a feedback response to reactivate the targeted pathway [36]. Targeting multiple pathways also helps in decreasing drug-induced toxicity by using sub-toxic doses in combination.
There have been many studies performed to investigate the role of Cox-2 and FoxM1 in tumorigenesis independently however there are only few studies where these molecules are studied together [37]. Therefore, in this study, we first investigated co-expression of Cox-2 and FoxM1 in CRC clinical samples followed by determining whether targeting of co-expression of FoM1 and Cox-2 can generate efficient anticancer effects in CRC cells both in vitro as well as in vivo models.

\section{Results}

\section{Evaluation of molecular expression of Cox-2 and FoxM1} in CRC tissues

Immunohistochemical analysis of Cox-2 expression was interpretable in $726 \mathrm{CRC}$ spots and the incidence of Cox-2 over-expression was found to be $60.6 \%$ (440/ 726). FoxM1 expression was interpretable in 719 CRC spots and the incidence of FoxM1 over-expression was found to be $50.3 \%(362 / 719)$. Cox-2 was seen predominantly in cytoplasmic compartment and FoxM1 expression was seen predominantly in the nuclear compartment. Coexpression of Cox-2 and FoxM1 was seen in 33.3 \% (232/ 697) of cases and were significantly associated with each other $(p=0.0115)$. Co-expression of Cox-2 and FoxM1 were found to be significantly associated with clinical parameters such as younger age $(p=0.0191)$ and mucinous histology $(p=0.0174)$ but were not associated with sex, or American Joint Committee on Cancer (AJCC) stage. Molecular association of this co-expression was seen with proliferative marker Ki-67 $(p=0.0004)$, p-AKT ( $p=$ $0.0214)$ and MMP-9 $(p=0.0116)$ (Table 1). No survival difference was seen between patients showing Cox-2 and FoxM1 co-expression and those with normal or reduced expression $(p=0.4796)$ (Table 1 and Additional file 1: Figure S1).

\section{Inhibition of Cox-2 and FoxM1 causes inhibition of cell viability in CRC cell lines}

In vitro, we initially sought to determine expression of Cox-2 and FoxM1 in a panel of CRC cell lines by immuno-blotting. We found that out of five CRC cell lines, only HT29 and Caco-2 had constitutive co-expression of Cox-2 and FoxM1 (Fig. 1a) therefore we selected these two cell lines in our study. We next determined the effect of Cox-2 inhibitor NS398 and FoxM1 inhibitor Thiostrepton [38] that has also been shown to possess proteasomal inhibition activity [39] on the expression of these proteins. At first, Caco-2 and HT29 cells were treated with 50 and $100 \mu \mathrm{M}$ NS398 for 48 h. NS398 treatment failed to downregulate the expression of FoxM1 in both the cell lines, even though, expression of Cox-2 was down-regulated and there was inactivation of AKT (Fig. 1b). This data was further confirmed by transfecting HT29 cells with specific siRNA targeted against Cox-2. As shown in Fig. 1c, similar 
Table 1 Correlation of Cox-2 \& Fox-M1 co-expression with clinico-pathological parameters in colorectal carcinoma $^{\text {a }}$

\begin{tabular}{|c|c|c|c|c|c|c|c|}
\hline & \multicolumn{2}{|l|}{ Total } & \multicolumn{2}{|l|}{ Both high } & \multicolumn{2}{|c|}{ Any-1-low } & \multirow[t]{2}{*}{$p$ value } \\
\hline & Number & Percent & Number & Percent & Number & Percent & \\
\hline Total number of cases & 697 & & 232 & 33.3 & 465 & 66.7 & \\
\hline \multicolumn{8}{|l|}{ Age } \\
\hline$\leq 50$ years & 229 & 32.9 & 90 & 39.3 & 139 & 60.7 & \multirow[t]{2}{*}{0.0191} \\
\hline$>50$ years & 468 & 67.1 & 142 & 30.3 & 326 & 69.7 & \\
\hline \multicolumn{8}{|l|}{ Sex } \\
\hline Male & 358 & 51.4 & 116 & 32.4 & 242 & 67.6 & \multirow[t]{2}{*}{0.6111} \\
\hline Female & 339 & 48.6 & 116 & 34.2 & 223 & 65.8 & \\
\hline \multicolumn{8}{|l|}{ Tumour Site } \\
\hline Left colon & 548 & 82.3 & 176 & 32.1 & 372 & 67.9 & \multirow[t]{2}{*}{0.5821} \\
\hline Right colon & 118 & 17.7 & 41 & 34.8 & 77 & 65.2 & \\
\hline \multicolumn{8}{|l|}{ Histological Type } \\
\hline Adenocarcinoma & 628 & 90.1 & 200 & 31.9 & 428 & 68.1 & \multirow[t]{2}{*}{0.0174} \\
\hline Mucinous Carcinoma & 69 & 9.9 & 32 & 46.4 & 37 & 53.6 & \\
\hline \multicolumn{8}{|l|}{ Tumour Stage } \\
\hline । & 80 & 12.2 & 25 & 31.3 & 55 & 68.7 & \multirow[t]{4}{*}{0.4160} \\
\hline$\|$ & 233 & 35.4 & 75 & 32.2 & 158 & 67.8 & \\
\hline III & 266 & 40.4 & 98 & 36.8 & 168 & 63.2 & \\
\hline IV & 79 & 12.0 & 22 & 27.8 & 57 & 72.2 & \\
\hline \multicolumn{8}{|l|}{ Differentiation } \\
\hline Well & 66 & 9.5 & 21 & 31.8 & 45 & 68.2 & \multirow[t]{3}{*}{0.3709} \\
\hline Moderate & 549 & 78.8 & 178 & 32.4 & 371 & 67.6 & \\
\hline Poor & 82 & 11.8 & 33 & 40.2 & 49 & 59.8 & \\
\hline \multicolumn{8}{|l|}{ Ki-67 } \\
\hline High & 590 & 87.7 & 209 & 35.4 & 381 & 64.6 & \multirow[t]{2}{*}{0.0004} \\
\hline Low & 83 & 12.3 & 14 & 16.9 & 69 & 83.1 & \\
\hline \multicolumn{8}{|l|}{ p-AKT } \\
\hline High & 454 & 72.3 & 169 & 37.2 & 285 & 62.8 & \multirow[t]{2}{*}{0.0214} \\
\hline Low & 174 & 27.7 & 48 & 27.6 & 126 & 72.4 & \\
\hline \multicolumn{8}{|l|}{ MMP-9 } \\
\hline High & 342 & 52.2 & 129 & 37.7 & 213 & 62.3 & \multirow[t]{2}{*}{0.0116} \\
\hline Low & 313 & 47.8 & 89 & 28.4 & 224 & 71.6 & \\
\hline \multicolumn{8}{|l|}{ Survival } \\
\hline OS 5 Years & & & & 73.6 & & 68.5 & 0.4796 \\
\hline
\end{tabular}

${ }^{a}$ Data were not available for (Tumor Site NA $\left.=31\right)$, (Tumor Stage NA $\left.=39\right)$, (Ki-67 NA $\left.=24\right),(p-A K T ~ N A=69)$ and $(M M P-9$ NA $=42)$

results were obtained where there was no effect on the expression of FoxM1 in CRC cell lines while the expression of Cox-2 decreased and there was in-activation of AKT following transfection with siRNA targeting Cox-2. In a separate experiment, CRC cell lines were treated with 5 and $10 \mu \mathrm{M}$ Thiostrepton for $48 \mathrm{~h}$ and immunoblotted with FoxM1, Cox-2, p-AKT and total AKT antibodies. The doses of Thiostrepton used have been previously shown to down-regulate expression of FoxM1 in other tumor cell lines without any off target effect or toxicity to normal peripheral blood mononuclear cells (PBMNC) [40, 41]. As shown in Fig. 1d, Thiostrepton treatment down-regulated expression of FoxM1 and Cox-2 and caused dephosphorylation of $\mathrm{AKT}$ at $10 \mu \mathrm{M}$ in both the cell lines. Similar results were obtained when CRC cell lines were transfected with siRNA targeted against FoxM1 for $48 \mathrm{~h}$ and immunoblotted with antibodies against FoxM1, Cox-2, p-AKT and total AKT (Fig.1e). These data suggest that FoxM1 is expressing upstream of Cox-2 and there is a link between FoxM1 and Cox-2 in CRC cells. Finally, we sought to 


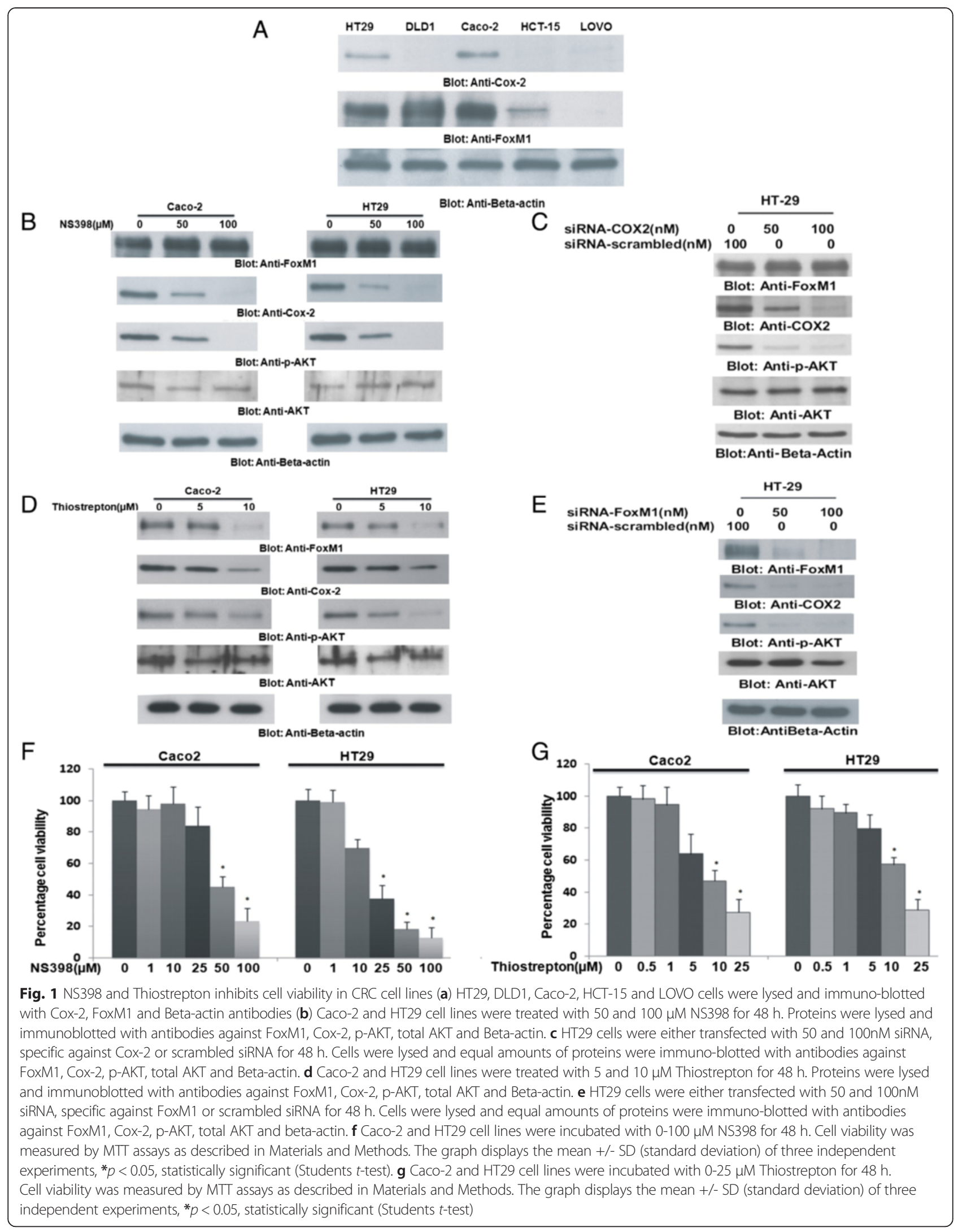


determine whether treatment of CRC cell lines with Cox-2 and FoxM1 inhibitors leads to inhibition of cell viability. Caco-2 and HT29 were cultured in the presence of 1, 10, 25, 50 and $100 \mu \mathrm{M}$ NS398 for $48 \mathrm{~h}$ and cell viability was assayed using MTT assay. As shown in Fig. 1f, there was a dose dependent inhibition of cell viability in both the cell lines that reached significance at $50 \mu \mathrm{M}$ for Caco- 2 and $25 \mu \mathrm{M}$ for HT29 respectively $(\mathrm{p}<0.05)$. Interestingly, this response was not seen in Cox-2 deficient DLD1 and LOVO cells up to doses of $100 \mu \mathrm{M}$ NS398 (Additional file 2: Figure S2 and Additional file 3: Figure S3). Similarly, the two cell lines were cultured in the presence of $0.5,1,5,10$ and $25 \mu \mathrm{M}$ Thiostrepton for $48 \mathrm{~h}$. Data revealed that there was also a dose dependent response to Thiostrepton treatment that reached statistical significance at $10 \mu \mathrm{M}$ for HT29 and Caco-2 cell lines respectively (Fig. 1b). In addition, there was partial response in FoxM1 negative cell line; LOVO as shown in Additional file 3: Figure S3. These data suggest that targeting Cox-2 and FoxM1 using specific inhibitors led to inhibition of cell viability in CRC cells.

\section{Synergistic activity of thiostrepton and NS398 in CRC cell lines}

As our data showed FoxM1 and Cox-2 co-expression was present in CRC, we hypothesized that targeting of
FoxM1 and Cox-2 expression together can lead to efficient cytotoxic effects in CRC cells. Therefore we sought to determine whether co-treatment of CRC cell lines with Thiostrepton, and NS398 at sub-toxic doses, can potentiate anticancer effects in CRC cells.

We conducted multiple experiments to determine the optimal doses that could be used in combination to inhibit cell viability, migration and colony formation and induce apoptosis in CRC cell lines. Using Chou and Talalay method [42], we found that $5 \mu \mathrm{M}$ Thiostrepton and 10uM NS398 in combination exerted maximum synergistic apoptotic response in HT29 and Caco-2 cells with combination index of 0.286 and 0.332 respectively (Fig. 2, Additional file 4: Table S1, Additional file 5: Table S2). Therefore, we first treated CRC cells with suboptimal doses of Thiostrepton $(5 \mu \mathrm{M})$ and NS398 $(10 \mu \mathrm{M})$ for various time points and found that the optimal synergistic response was detected at $48 \mathrm{~h}$ following treatment (Additional file 6: Figure S4). Therefore, we treated Caco2 and HT29 cells with combination of NS398 and Thiostrepton for $48 \mathrm{~h}$ and assessed the cell viability by MTT assay. As shown in Fig. 3a, neither Thiostrepton nor NS398 alone could inhibit cell viability, however, cotreatment with Thiostrepton and NS398 led to significant inhibition of cell viability in CRC cells $(\mathrm{p}<0.01)$. Next, we determined whether combination of Thiostrepton and
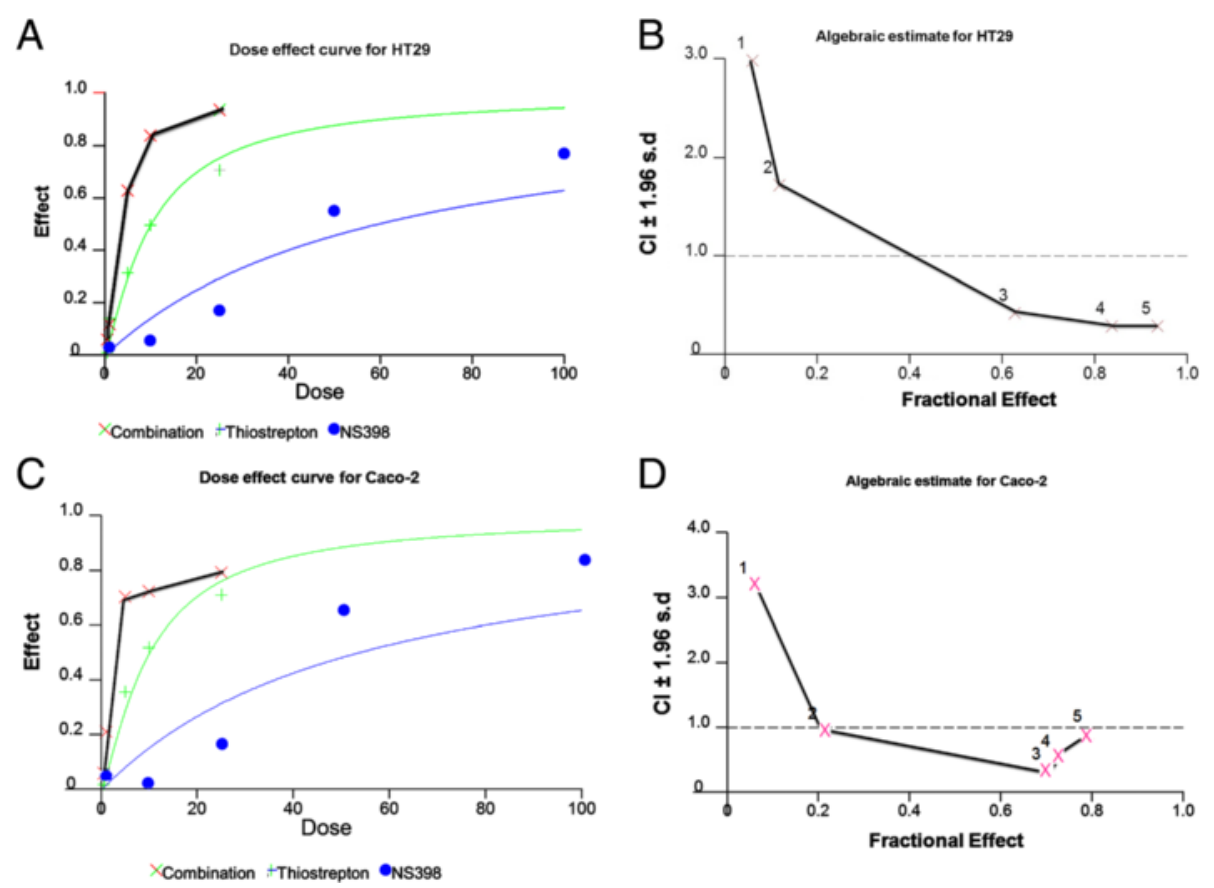

Fig. 2 Calculation of optimal doses required for co-treatment of CRC cells by Thiostrepton and NS398. HT29 and Caco-2 cells were treated with $0.5,1.0,5.0,10$ and $25 \mu \mathrm{M}$ Thiostrepton or 1.0, 10, 25, 50 and $100 \mu \mathrm{M} \mathrm{NS398}$ alone or in different combinations to calculate the Synergistic apoptotic response of Thiostrepton and NS398 for $48 \mathrm{~h}$ and dose effect ( $\mathbf{a}$ and $\mathbf{c}$ ) and Fractional effect (b and $\mathbf{d})$ graphs were generated using Calcusyn software. Apoptotic response analysis was done as mean \pm SD values normalized to control. Combination indices were calculated using Chou and Talalay methodology 


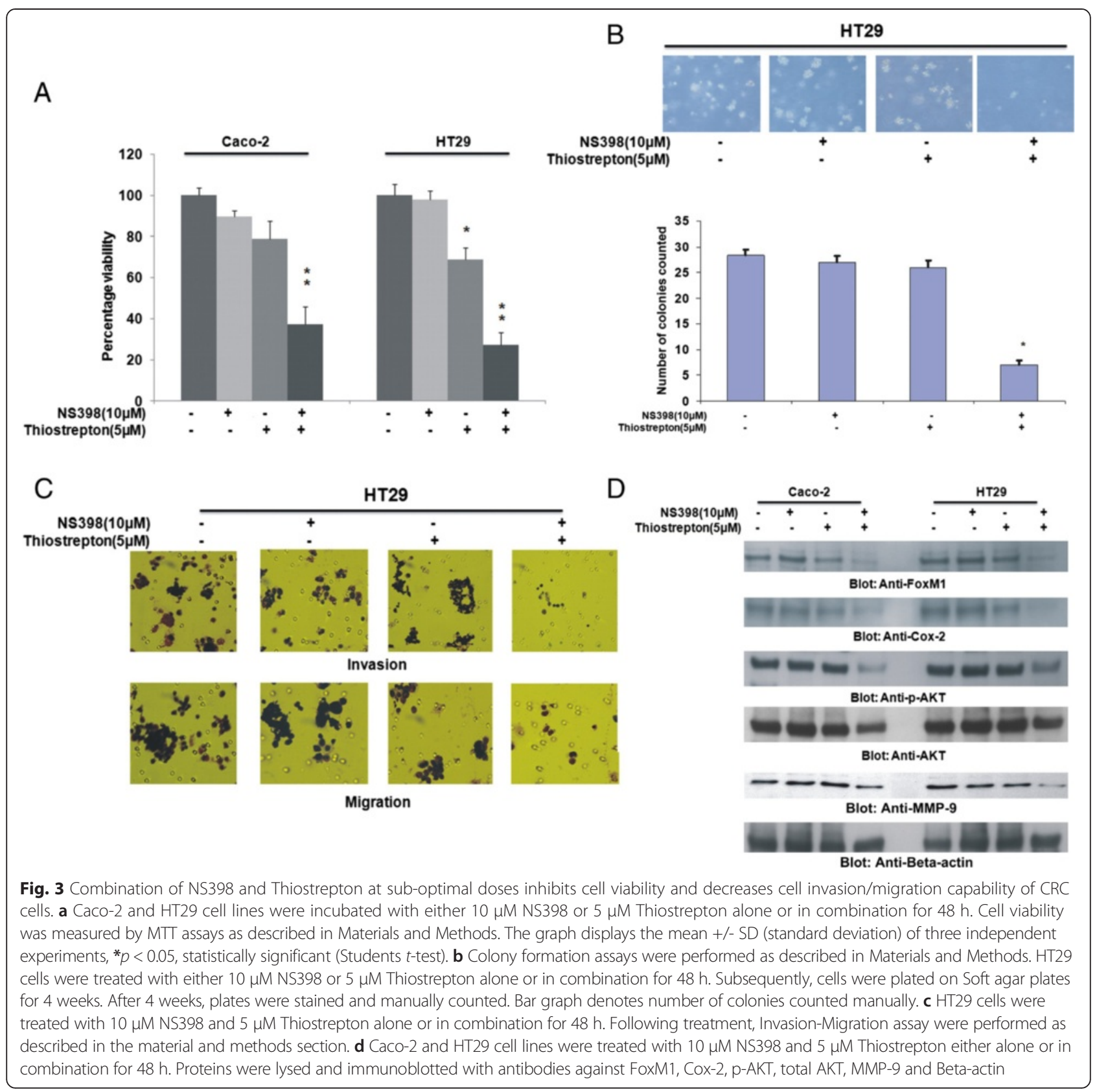

NS398 could inhibit colony formation in CRC cells. As shown in Fig. 3b, there was significant inhibition in colony formation in HT29 cells as compared to treatment alone. Combination of Thiostrepton and NS398 also inhibited cell invasion and migration in HT29 cells as compared to treatment alone (Fig. 3c). We finally investigated whether co-treatment of CRC cells with sub-optimal doses of Thiostrepton and NS398 could inhibit expression of FoxM1, Cox-2 and MMP-9 by immunoblotting. As shown in Fig. 3d, combination treatment of Thiostrepton and NS398 successfully down-regulated expression of FoxM1, Cox-2 and MMP-9 and inactivated p-AKT without disrupting the expression of total AKT in Caco-2 and HT29 cell lines. These data indicate that co-treatment with Thiostrepton and NS398 synergistically inhibits cell viability and migratory properties of CRC cells. Finally, Cox-2 deficient cell line; DLD1 cells and Cox-2 and FoxM1 deficient cell line; LOVO did not show any synergistic response when treated with combination of $5 \mu \mathrm{M}$ Thiostrepton and $10 \mu \mathrm{M}$ NS398 and the cell inhibition detected were due to Thiostrepton treatment alone (Additional file 7: Table S3 and Additional file 8: Table S4) confirming specificity of NS398 and Thiostrepton treatment against Cox-2 and FoxM1expression. 


\section{Co-treatment of CRC cells with thiostrepton and NS398} induces apoptosis via mitochondrial apoptotic pathway For efficient apoptosis to occur, the mitochondrial apoptotic pathway needs to be activated. Bax, a pro-apoptotic $\mathrm{BH} 3$ domain only protein of the Bcl-2 family [43] is the first protein that undergoes conformational changes for the mitochondrial apoptotic pathway to be activated [44]. To investigate the effect of co-treatment with Thiostrepton and NS398 on activation of the mitochondrial pathway, we treated HT29 cells for various time periods and examined the conformational changes in Bax protein by immuno-precipitation. As shown in Fig. 4a, conformationally changed Bax was detected after $2 \mathrm{~h}$, peaked within $16 \mathrm{~h}$ and then decreased at $24 \mathrm{~h}$ of treatment with $5 \mu \mathrm{M}$ Thiostrepton and $10 \mu \mathrm{M}$ NS398. Once Bax is conformationally changed, it causes changes in the mitochondrial membrane potential. To investigate this, we treated Caco2 and HT29 cells with either Thiostrepton or NS398 alone or in combination for $48 \mathrm{~h}$ and examined the change in mitochondrial membrane potential by flow cytometry. Neither Thiostrepton nor NS398 could affect the mitochondrial membrane potential alone however when combination of both inhibitors were used together, there was an increase in cells undergoing mitochondrial membrane damage as depicted by green bars (Fig. 4b). Once there are changes in the mitochondrial membrane potential, there is release of cytochrome into the cytosole (Data not shown) leading to activation and cleavage of downstream caspases. To determine this, we treated HT29 cells with NS398 $(10 \mu \mathrm{M})$, Thiostrepton $(5 \mu \mathrm{M})$ and a combination of the two for $48 \mathrm{~h}$ and examined cleavage of caspase-9, caspase3 and PARP by immunoblotting. Caspase- $9,-3$ and PARP were cleaved in cells that were treated with combination of Thiostrepton and NS398 (Fig. 4c). Once the downstream caspases are activated and cleaved, it leads to cell death via apoptosis. To confirm this, we visualized cells under an Olympus fluorescent microscope using a longpass filter after 48 h treatment with NS398, Thiostrepton or a combination of two inhibitors after staining them with $50 \mu \mathrm{M}$ calcein AM and $8 \mu \mathrm{M}$ ethidium homodimer. As shown in Fig. $4 d$, there were more green stained cells depicting live cells in samples treated with Thiostrepton or NS398 alone. The cells stained red representing dead cells in sample that was treated with combination of Thiostrepton and NS398. Apoptosis was further confirmed by annexinV/PI dual staining that was investigated by flow cytometry. These set of data confirm that combination of Thiostrepton and NS398 treatment induces apoptosis in CRC cells via activation of mitochondrial apoptotic pathway.

Inhibition HT29 xenografts by combinational treatment of thiostrepton and NS398 in nude mice

The synergistic effect of the combination of Thiostrepton and NS398 in vitro suggested that this combination would likely to be effective in tumor xenografts in vivo. Therefore, we sought to determine whether co-treatment of Thiostrepton with NS398 potentiated the inhibition of CRC cell line generated xenograft tumor in nude mice. For xenograft study, mice were inoculated subcutaneously into the right abdominal quadrant with10 million HT29 cells in $200 \mu \mathrm{l}$ PBS. After 1 week of inoculation, mice were randomly assigned into four groups: The first group received DMSO as control vehicle while the other three groups received NS398 (15 mg/kg), Thiostrepton (150 $\mathrm{mg} / \mathrm{kg}$ ) and combination of $15 \mathrm{mg} / \mathrm{kg}$ NS398 and $150 \mathrm{mg} / \mathrm{kg}$ Thiostrepton, injected twice weekly, intraperitoneally respectively. After 5 weeks treatment, mice were sacrificed and tumors were collected. As shown in Fig. 5a, there was significant regression of tumor volume at the end of second week in the group of animal treated with Thiostrepton and NS398. Neither Thiostrepton nor NS398 alone resulted in significant inhibition of xenograft tumors. A significant reduction in tumor weight (Fig. 5b) was also observed in mice treated with Thiostrepton and NS398 $(\mathrm{p}<001)$. We also visualized images of the tumor, postnecropsy and found that there was significant shrinkage in the size of the tumor following treatment with combination of Thiostrepton and NS398 as compared to treatment alone (Fig. 5c). Finally, we analyzed the status FoxM1, Cox-2, p-AKT, total AKT and caspase-3 in HT29 xenograft at the end of study. As shown in Fig. $5 \mathrm{~d}$, the level of FoxM1, Cox-2, MMP-9 and caspase-3 were remarkably deceased along with in-activation of AKT in tumors of mice co-treated with Thiostrepton and NS398, compared to vehicle, Thiostrepton and NS398 alone treatment. Our data indicates that co-treatment with Thiostrepton and NS398 augmented antitumor effects in HT29 cell xenografts in Nude mice.

\section{Discussion}

In this study, we have investigated the role of Cox-2 and FoxM1 co-expression in a large cohort of 770 Middle Eastern CRC in a tissue microarray format to determine the protein expression by immunohistochemistry. It has been previously shown that Cox-2 and FoxM1 expression are associated with a poor prognosis in CRC $[45,46]$. In this study, we found a significant association between FoxM1 and Cox-2 over-expression in Middle Eastern CRC samples $(p=0.0115)$. Co-expression of FoxM1 and Cox-2 was also found to be associated with an aggressive phenotype that was characterized by younger age ( $p=$ $0.0191)$, proliferative marker Ki-67 (0.0004), MMP-9 ( $p=$ $0.0116)$ and activation of AKT $(p=0.0214)$. These data reiterates the point that targeting these two molecules simultaneously using small molecular inhibitors may be more beneficial for the management of this aggressive phenotype of CRC when compared to treatment with single agent. 


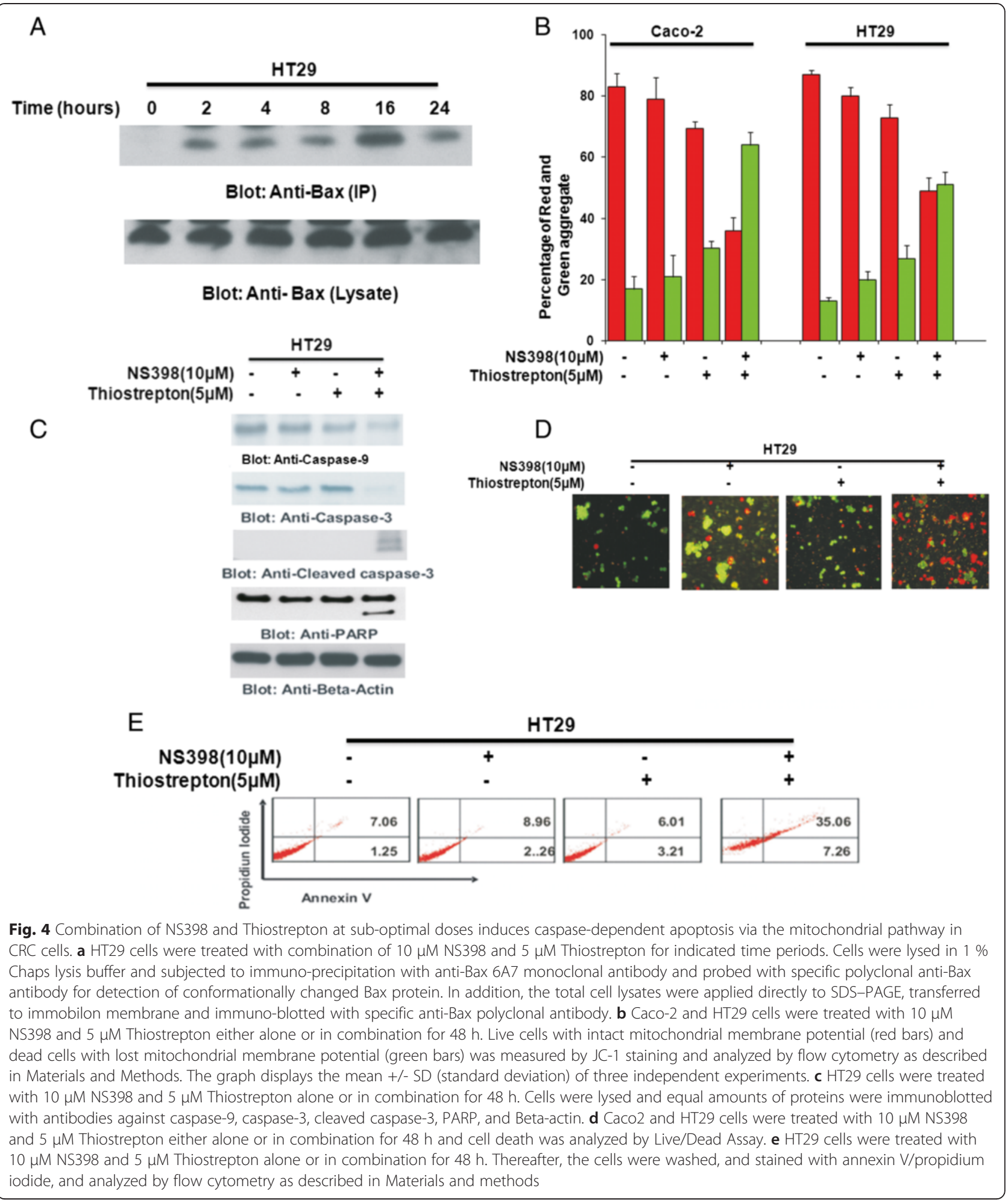

Cross talk between survival pathways is slowly emerging as one of the leading causes of drug resistance to small molecular inhibitors for the treatment of cancer. After an initial response to the treatment, resistance to therapy quickly develops due to re-activation of the target molecule either by up-stream pro-survival pathway molecules or negative feed-back mechanism by down-stream molecules. To counteract this resistance, recent studies have shown targeting multiple pro-survival molecules of different survival pathways that are associated 


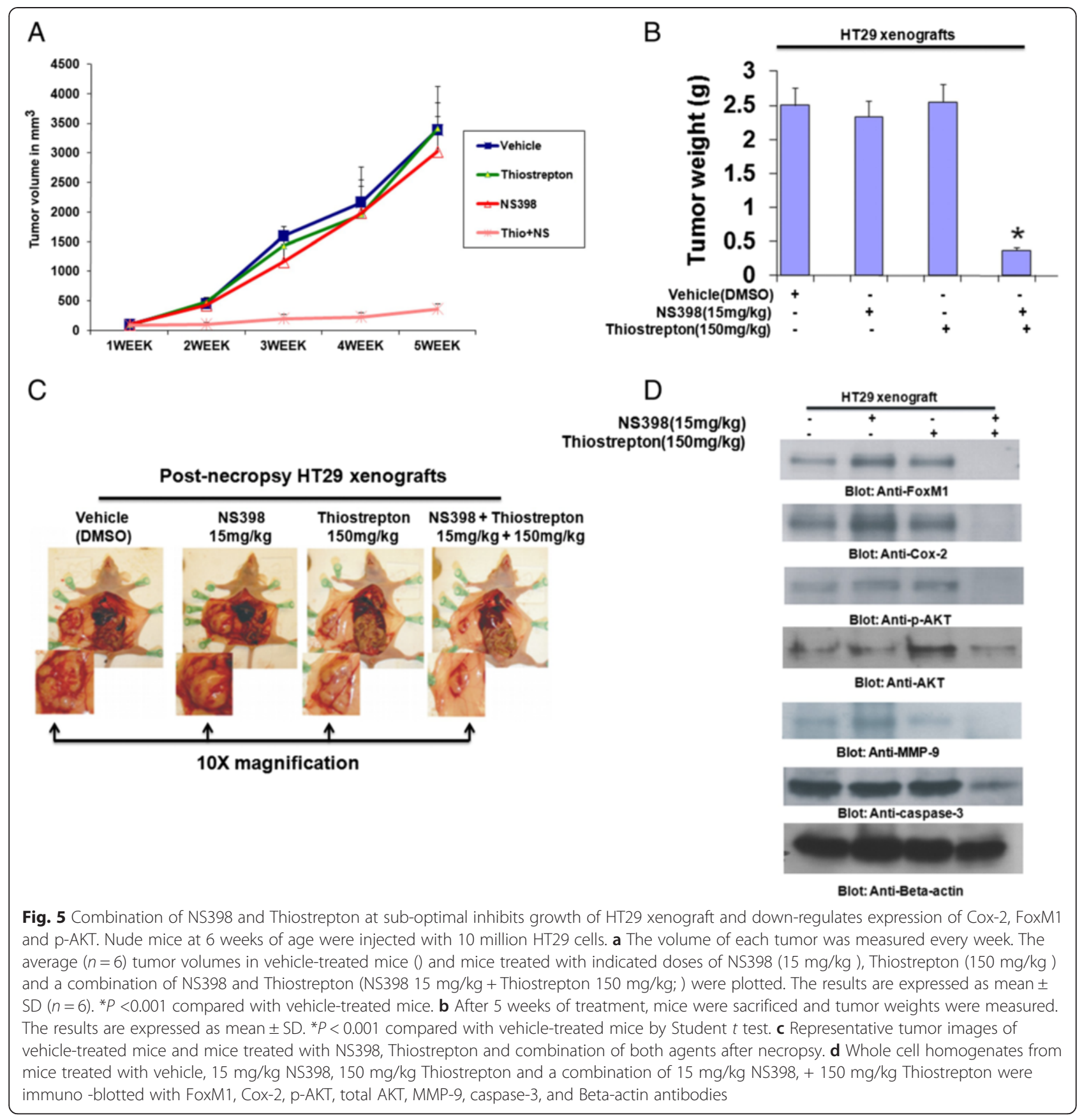

with each other with combination of specific inhibitors simultaneously is more beneficial than treatment with single agent alone $[47,48]$. Combination treatment is also beneficial because the dose of each drug is considerably decreased when used in combination thereby reducing the chances of toxicity to normal cells. Therefore, the role of single agent treatment with molecular inhibitors is diminishing and targeting various cancers with multiple inhibitors is on the rise.

A strong association between expression of FoxM1 and Cox-2 with MMP-9 expression has been reported in a number of cancers $[31,49,50]$. Previously, it has also been reported in lung cancer that transcriptional depletion of FoxM1 expression can cause reduced Cox- 2 expression and on the other hand, induced over-expression of FoxM1 protein can increase Cox-2 promoter activity [37]. These findings are in conco rdance with our data where pharmacological and transcriptional inhibition of FoxM1 expression down-regulates the expression of Cox-2. Furthermore, our data also demonstrates that depletion of Cox-2 expression does not affect FoxM1 expression suggesting that FoxM1 is functional upstream of Cox-2. Combined 
targeting of Cox-2 and FoxM1 with pharmacological inhibitors also depletes colony formation as well as invasive and migratory capabilities of CRC cells in vitro via down-regulation of MMP-9 thereby indicating the utility of combined targeting of these molecules for inhibition of metastasis in CRC cells.

Combined targeting of Cox-2 and FoxM1 not only inhibits the invasive and migratory capability of CRC cells, they also cause inhibition of cell viability and induction of apoptosis. This effect occurs via inactivation of an important survival molecule; $\mathrm{p}$-AKT, that plays an important role in the survival of cancer cells and is found to be constitutively activated in various cancers [51]. Our data showed that dephosphorylation of AKT led to activation of the mitochondrial apoptotic pathway initiated by Bax conformational changes and translocation to the mitochondrial membrane, thereby leading to changes in the mitochondrial membrane potential and finally activation and cleavage of caspases. Once caspases are activated, there is cleavage of PARP; an essential enzyme that is required for repairing single stranded breaks in DNA [49] and is a hallmark of cells undergoing apoptosis. Our in vivo studies further validate our hypothesis that cotreatment of mice bearing palpable CRC xenograft with Thiostrepton and NS398 leads to regression of tumor growth via down-regulation of FoxM1, Cox-2, MMP-9, inactivation of AKT and cleavage of caspase- 3 which is consistent with our in vitro findings.

\section{Conclusions}

Altogether, we found that $33.3 \%$ of CRC clinical samples co-express Cox-2 and FoxM1 and this sub-group is associated with an aggressive phenotype. Therefore, our data highlights the importance of co-targeting of deregulated survival pathways (Cox-2 and FoxM1) in CRC cells can lead to anticancer effects. Our data showed that combination treatment of CRC cells with sub-optimal doses of Thiostrepton and NS398 caused functional inhibition of Cox-2 and FoxM1 simultaneously. Even though, Thiostrepton and NS398 have been previously shown to be effective in suppressing growth and inducing apoptosis in CRC cells at higher concentrations [31, 52], this study emphasizes the importance of targeting multiple survival molecules with sub-optimal doses of Thiostrepton and NS398 to successfully inhibit cell growth, invasion, migration and induce apoptosis in CRC. Further studies are warranted to further investigate the utility of combination treatment with Thiostrepton and NS398 for the treatment of CRC in clinical settings.

\section{Material and methods}

\section{Patient selection and tissue microarray construction}

Seven hundred and seventy patients with CRC diagnosed between 1990 and 2011 were selected from King Faisal
Specialist Hospital and Research Centre. Clinical and histopathological data were available for all these patients. Colorectal Unit, Department of Surgery, provided long-term follow-up data. Patients with colon cancer underwent surgical colonic resection and rectal cancer underwent anterior resection or abdominoperineal resection. Majority of node positive colon cancers received 5 -fluorouracil based adjuvant chemotherapy. A vast majority of the rectal cancers received radiotherapy alone or chemo-radiotherapy prior to surgery followed by adjuvant chemotherapy after surgery. Tissue microarrays were constructed from formalin-fixed, paraffin-embedded colorectal carcinoma specimens as described previously [53]. Institutional Review Board (IRB) of the King Faisal Specialist Hospital \& Research Centre approved the study (NSTIP 10-BIO-959-20 and RAC 2140 005).

\section{Immunohistochemistry (IHC)}

TMA slides were processed and stained manually as described previously [54]. Sections were deparaffinized in xylene and rehydrated through graded alcohol to water. Antigen retrieval was done in a Pascal Pressure cooker at $120{ }^{\circ} \mathrm{C}$ for 8 min using the Dako Retrieval solution, pH 6 (S2369; Dako Cytomation, Copenhagen, Denmark). Endogenous peroxidase activity was blocked by incubating the slides in $3 \% \mathrm{H}_{2} \mathrm{O}_{2}$ in water for $30 \mathrm{~min}$ at room temperature. Sections were incubated in $1 \%$ BSA for 30 min then wiped off and dilution of Cox-2 and FoxM1 was applied on the slides and incubated overnight at room temperature. Subsequently sections were incubated with Envision + secondary antibody for $1 \mathrm{~h}$ at room temperature and visualization was done using the liquid $\mathrm{DAB}+$ substrate chromogen system. Only fresh cut slides were stained simultaneously to minimize the influence of slide ageing and maximize repeatability and reproducibility of the experiment. Details of primary antibodies used, dilutions, cut-off and incidences of positive cases are listed in Additional file 9: Table S5. H-score was used for categorizing the expression of Cox-2 and FoxM1. Each TMA spot was assigned an intensity score from 0 to 3 (I0, I1-I3) and proportion of the tumor staining for that intensity was recorded in $5 \%$ increments from 0 to 100 (P0, P1-P3). A final $\mathrm{H}$ score (range, 0-300) was obtained by adding the sum of scores obtained for each intensity I and proportion of area stained. X-tile plots were constructed for assessment of biomarker and optimization of cutoff points based on outcome, as described previously [55]. The CRCs were stratified into two groups based on X-tile plots: one with complete absence or reduced staining and the other with overexpression. For Ki-67 cut-off of $\geq 50 \%$ nuclear staining was used and for p-AKT intensity score $2+/ 3+$ was considered as positive. 


\section{Statistical analysis}

Contingency table analysis and $\chi^{2}$ tests were used to study relationship between clinicopathological variables and gene amplification. The limit of significance for all analyses was defined as a $P$ value of 0.05 ; two-sided tests were used in all calculations. The JMP 10.0 software package (SAS Institute, Cary, NC) was used for data analyses.

\section{Reagents and antibodies}

Thiostrepton (FoxM1 selective inhibitor) [56] was purchased from Tocris Cookson Inc (Ellisville, MO). NS398 (COX-2 inhibitor) was purchased from Caymen chemical company, (Ann Arbor, MI). Antibodies against cleaved caspase-3, Cox-2, AKT and p-AKT antibodies were purchased from Cell Signaling Technologies (Beverly, MA). FoxM1, Bax, Beta-actin, caspase-3 and poly (ADP) ribose polymerase (PARP) antibodies were purchased from Santa Cruz Biotechnology, Inc. (Santa Cruz, CA). MMP-9 antibodies were purchased from Anespec, (San Jose, CA). Annexin V/PI kit was purchased from Molecular Probes (Eugene, OR, USA).

\section{Cell culture}

HT29, DLD1, LOVO, HCT-15 and Caco-2 were obtained from Deutsche Sammlung von Mikroorganismen und Zellkulturen (DSMZ), Braunschweig, Germany. All cell lines were tested for immunological markers and cytogenetics. The cell lines were also fingerprinted and species was confirmed by IEF of AST, MDH and NP. Cells were cultured in RPMI 1640 medium supplemented with $10 \%$ (vol/vol) fetal bovine serum, $100 \mathrm{U} /$ $\mathrm{ml}$ Penicillin and $100 \mathrm{U} / \mathrm{ml}$ Streptomycin at $37^{\circ} \mathrm{C}$ in humidified atmosphere containing $5 \% \mathrm{CO}_{2}$. All the experiments were performed in RPMI-1640 containing $5 \%$ fetal bovine serum.

\section{Cell growth studies by 3-(4,5-dimethylthiazol-2-yl)-2,5-} diphenyltetrazolium bromide (MTT) assays

$10^{4}$ cells were incubated in triplicate in a 96-well plate in a final volume of $0.2 \mathrm{ml}$ for $48 \mathrm{~h}$ at $37^{\circ} \mathrm{C}$. Cell viability assay using MTT was performed as described previously [57].

\section{Live dead assay}

To determine cell death, Live-Dead assay (Invitrogen, Eugene, OR) was used as described by the manufacturer. HT29 and Caco-2 cells were treated either alone with NS398, and Thiostrepton or in combination as described in the legends. Following incubation for $48 \mathrm{~h}$, cells were suspended in $1 \mathrm{ml}$ PBS containing $50 \mathrm{mM}$ calcein AM and $8 \mathrm{mM}$ ethidium homodimer and cells were incubated in the dark for $20 \mathrm{~min}$. $50 \mu \mathrm{l}$ of suspension was transferred on slides and visualized under an Olympus fluorescent microscope using a longpass filter.

\section{Soft agar colony assay}

Soft agar colony experiments were performed according to the manufacturer's protocol (Cheminon International, Temecula, CA, USA). Briefly, after treatment of cells with NS398, Thiostrepton or a combination of the two inhibitors for $48 \mathrm{~h}, 2500$ cells were plated in $0.5 \mathrm{ml}$ culture medium containing $0.4 \%(\mathrm{v} / \mathrm{v})$ top agar and $20 \%$ fetal bovine serum (FBS) layered over a basal layer of $0.8 \%(\mathrm{v} / \mathrm{v})$ agar and $20 \%$ FBS with culture medium and allowed to grow for 4 weeks as described previously (our REF). Following 4 weeks incubation, cells were stained at a final concentration of $1 \mathrm{mg} / \mathrm{ml}$ cell stain solution that was supplied with the kit.

\section{Cell invasion and migration assay}

Cell invasion and migration assay were performed using 24-well Transwell Permeable Supports with 8-1M pores (Corning, Lowell, MA). Briefly, after treatment of cells with NS398, Thiostrepton or a combination of the two inhibitors for $48 \mathrm{~h}$, cells were harvested, counted again and $1.25 \times 105$ cells were suspended in serum-free medium and seeded into Transwell inserts either uncoated (for migration assay) or coated (for invasion assay) with growth factor-reduced Matrigel (BD Biosciences, Bedford, MA). Bottom wells were filled with complete media for $24 \mathrm{~h}$. After incubation of $24 \mathrm{~h}$, filters containing the cells were stained with Diff-Quick stain set (Fisher Scientific, Pittsburg, PA), photographed under a fluorescent microscope and manual cell counts were obtained [41].

\section{Gene Silencing using siRNA}

FoxM1 siRNA, Cox-2 siRNA and Scrambled control siRNA were purchased from Qiagen (Valencia, CA, USA). Cells were transfected using Lipofectamine 2000 (Invitrogen, Carlsbad, CA) for $6 \mathrm{~h}$ following which the lipid and siRNA complex was removed and fresh growth medium was added. Cells were lysed $48 \mathrm{~h}$ after transfection and specific protein levels were determined by Western Blot analysis with specific antibodies.

\section{Annexin V/PI dual staining}

HT29 and Caco-2 cells were treated either with NS398, Thiostrepton or in combination as described in the legends. For detection of apoptosis, cells were harvested and percentage apoptosis was measured by flow cytometry after staining with flourescein-conjugated annexin- $\mathrm{V}$ and propidium iodide (PI) (Molecular probes, Eugene, OR) [58].

\section{Measurement of mitochondrial membrane potential}

Cells were treated with NS398 and Thiostrepton as described in the legends for $48 \mathrm{~h}$, washed twice with PBS, and re-suspended in mitochondrial incubation buffer. 
JC1 staining and flow cytometry were done as described previously.

\section{Cell lysis and immunoblotting}

Cells were lysed as previously described [44]. Proteins were immunoblotted with different antibodies and visualized by the enhanced chemiluminescence (Amersham, Piscataway, NJ) method.

\section{Detection of Bax conformational changes}

Detection of Bax conformation was performed as previously described [44]. In brief, HT29 cells were treated with combination of $10 \mu \mathrm{M}$ NS398 and $5 \mu \mathrm{M}$ Thiostrepton for indicated time periods after which proteins were lysed and immunoblotted using N20 Bax polyclonal antibody.

\section{Animals and xenograft study}

Six weeks old nude mice were obtained from Jackson Laboratories (Maine, USA) and maintained in a pathogen free animal facility at least 1 week before use. All animal studies were done in accordance with institutional guidelines. For Xenograft study, mice were inoculated subcutaneously into the right abdominal quadrant with $10 \times 10^{6}$ cells of HT29 in $200 \mu \mathrm{L}$ PBS. After 1 week, mice were randomly assigned into four groups: The first group received DMSO. The three groups received N398 (15 mg/ $\mathrm{kg})$, Thiostrepton $(150 \mathrm{mg} / \mathrm{kg})$ and combination of $15 \mathrm{mg} /$ kg NS398 and $150 \mathrm{mg} / \mathrm{kg}$ Thiostrepton, intra-peritoneally respectively. Mice were given these drugs twice weekly. The body weight and tumor volume of each mouse was monitored weekly. The tumor volume was measured as described previously [31]. After 5 weeks treatment, mice were sacrificed and individual tumors were weighed, then snap-frozen in liquid nitrogen for storage.

\section{Additional files}

Additional file 1: Figure S1. Kaplan-Meier survival curve of CRC cases showing co-expression of Cox-2 and FoxM1 as compared to cases with normal or reduced expression $(p=0.4796)$.

Additional file 2: Figure S2. DLD1 cells were incubated with 0-100 $\mu \mathrm{M}$ NS398 for 48 h. Cell viability was measured by MTT assays as described in Materials and Methods. The graph displays the mean +/- SD (standard deviation) of three independent experiments, ${ }^{*} p<0.05$, statistically significant (Students $t$-test).

Additional file 3: Figure S3. LOVO cells were incubated with 0-100 $\mu \mathrm{M}$ NS398, 0-25 $\mu \mathrm{M}$ Thiostrepton or a combination of both drugs for $48 \mathrm{~h}$. Cell viability was measured by MTT assays as described in Materials and Methods. The graph displays the mean +/- SD (standard deviation) of three independent experiments, ${ }^{*} p<0.05$, statistically significant (Students $t$-test).

Additional file 4: Table S1. Combination Index calculation using Chou and Talalay method in Caco-2 cell line.

Additional file 5: Table S2. Combination Index calculation using Chou and Talalay method in HT29 cell line.

Additional file 6: Figure S4. (A and B) Caco-2 (A) and HT29 (B) cells were incubated with either $10 \mu \mathrm{M}$ NS398, $5 \mu \mathrm{M}$ Thiostrepton or a combination of both drugs for indicated time points. Cell viability was measured by MTT assays as described in Materials and Methods. The graph displays the mean +/- SD (standard deviation) of three independent experiments, ${ }^{*} p<0.05$, statistically significant (Students $t$-test).

Additional file 7: Table S3. Combination Index calculation using Chou and Talalay method in DLD1 cell line.

Additional file 8: Table S4. Combination Index calculation using Chou and Talalay method in LOVO cell line.

Additional file 9: Table S5. Details of primary antibodies used in the study.

\section{Competing interests}

The authors declare that they have no competing interests.

\section{Authors' contributions}

MA performed in vitro and in vivo experiments. ARH performed experiments and helped in writing the manuscript. AKS analyzed the data. SU helped in writing the manuscript. NAS, MAA and FAD collected and analyzed the clinical data. SB performed all the immunohistochemistry experiments. KSA designed and supervised the execution of the study and wrote the paper All authors read and approved the final manuscript.

\section{Acknowledgements}

We would like to acknowledge the efforts of Dr. Sally Al Abdulmohsen, Saravanan Thangavel and Saeeda Ahmed for their technical assistance.

\section{Grant support}

This study was supported by King AbdulAziz Centre for Science and Technology with grant number NSTIP10-BIO959-20.

\section{Author details}

${ }^{1}$ Human Cancer Genomic Research, Research Center, Riyadh, Saudi Arabia. ${ }^{2}$ Department of Surgery, Colorectal unit, Riyadh, Saudi Arabia. ${ }^{3}$ Department of Pathology, King Faisal Specialist Hospital and Research Center, Riyadh, Saudi Arabia. ${ }^{4}$ Department of Surgery, Security Forces Hospital, Riyadh, Saudi Arabia. ${ }^{5}$ Al-Faisal University, Riyadh, Saudi Arabia.

Received: 18 March 2015 Accepted: 1 July 2015

Published online: 10 July 2015

\section{References}

1. Ross JS, Torres-Mora J, Wagle N, Jennings TA, Jones DM. Biomarker-based prediction of response to therapy for colorectal cancer: current perspective. Am J Clin Pathol. 2010;134:478-90.

2. Chan AT, Giovannucci EL. Primary prevention of colorectal cancer. Gastroenterology. 2010;138:2029-43.

3. Imamura Y, Lochhead P, Yamauchi M, Kuchiba A, Qian ZR, Liao X, et al. Analyses of clinicopathological, molecular, and prognostic associations of KRAS codon 61 and codon 146 mutations in colorectal cancer: cohort study and literature review. Mol Cancer. 2014;13:135.

4. Worthley DL, Leggett BA. Colorectal cancer: molecular features and clinical opportunities. Clin Biochem Rev. 2010;31:31-8.

5. Segal NH, Saltz LB. Evolving treatment of advanced colon cancer. Annu Rev Med. 2009;60:207-19.

6. Meyer-Kirchrath J, Schror K. Cyclooxygenase-2 inhibition and side-effects of non-steroidal anti-inflammatory drugs in the gastrointestinal tract. Curr Med Chem. 2000;7:1121-9.

7. Zhu YM, Azahri NS, Yu DC, Woll PJ. Effects of COX-2 inhibition on expression of vascular endothelial growth factor and interleukin-8 in lung cancer cells. BMC Cancer. 2008;8:218.

8. Zhu Z, Zhong S, Shen Z. Targeting the inflammatory pathways to enhance chemotherapy of cancer. Cancer Biol Ther. 2011;12:95-105.

9. Anto RJ, Mukhopadhyay A, Shishodia S, Gairola CG, Aggarwal BB. Cigarette smoke condensate activates nuclear transcription factor-kappaB through phosphorylation and degradation of IkappaB (alpha): correlation with induction of cyclooxygenase-2. Carcinogenesis. 2002;23:1511-8.

10. Howe LR. Inflammation and breast cancer. Cyclooxygenase/prostaglandin signaling and breast cancer. Breast Cancer Res. 2007;9. 
11. Uddin S, Ahmed M, Hussain A, Assad L, Al-Dayel F, Bavi P, et al. Cyclooxygenase-2 inhibition inhibits PI3K/AKT kinase activity in epithelial ovarian cancer. Int J Cancer. 2010;126:382-94.

12. Moreira L, Castells A. Cyclooxygenase as a target for colorectal cancer chemoprevention. Curr Drug Targets. 2011;12:1888-94.

13. Krawczyk-Rusiecka K, Wojciechowska-Durczynska K, Cyniak-Magierska A, Adamczewski Z, Galecka E, Lewinski A. COX-2 expression in papillary thyroid carcinoma (PTC) in cytological material obtained by fine needle aspiration biopsy (FNAB). Thyroid Res. 2011;4:3

14. Kim SJ, Rabbani ZN, Dong F, Vollmer RT, Schreiber EG, Dewhirst MW, et al. Phosphorylated epidermal growth factor receptor and cyclooxygenase-2 expression in localized non-small cell lung cancer. Med Oncol. 2010;27:91-7

15. Avivi D, Moshkowitz M, Detering E, Arber N. The role of low-dose aspirin in the prevention of colorectal cancer. Expert Opin Ther Targets. 2012;16 Suppl 1:S51-62.

16. Lo-Ciganic WH, Zgibor JC, Bunker CH, Moysich KB, Edwards RP, Ness RB. Aspirin, nonaspirin nonsteroidal anti-inflammatory drugs, or acetaminophen and risk of ovarian cancer. Epidemiology. 2012;23:311-9.

17. McCarty MF. Minimizing the cancer-promotional activity of cox-2 as a central strategy in cancer prevention. Med Hypotheses. 2012;78:45-57.

18. Printz C. Celecoxib may prevent lung cancer. Cancer. 2012;118:3.

19. Carlsson P, Mahlapuu M. Forkhead transcription factors: key players in development and metabolism. Dev Biol. 2002;250:1-23.

20. Kaestner $\mathrm{KH}$, Knochel W, Martinez DE. Unified nomenclature for the winged helix/forkhead transcription factors. Genes Dev. 2000;14:142-6.

21. Wang X, Kiyokawa H, Dennewitz MB, Costa RH. The Forkhead Box m1b transcription factor is essential for hepatocyte DNA replication and mitosis during mouse liver regeneration. Proc Natl Acad Sci U S A. 2002;99:16881-6.

22. Kalin TV, Wang IC, Ackerson TJ, Major ML, Detrisac CJ, Kalinichenko W, et al. Increased levels of the FoxM1 transcription factor accelerate development and progression of prostate carcinomas in both TRAMP and LADY transgenic mice. Cancer Res. 2006;66:1712-20.

23. Krupczak-Hollis K, Wang X, Kalinichenko W, Gusarova GA, Wang IC, Dennewitz MB, et al. The mouse Forkhead Box m1 transcription factor is essential for hepatoblast mitosis and development of intrahepatic bile ducts and vessels during liver morphogenesis. Dev Biol. 2004;276:74-88.

24. Ye H, Holterman AX, Yoo KW, Franks RR, Costa RH. Premature expression of the winged helix transcription factor $\mathrm{HFH}-11 \mathrm{~B}$ in regenerating mouse liver accelerates hepatocyte entry into $S$ phase. Mol Cell Biol. 1999;19:8570-80.

25. Wonsey DR, Follettie MT. Loss of the forkhead transcription factor FoxM1 causes centrosome amplification and mitotic catastrophe. Cancer Res. 2005:65:5181-9.

26. Kalinichenko W, Major ML, Wang X, Petrovic V, Kuechle J, Yoder HM, et al. Foxm 1b transcription factor is essential for development of hepatocellular carcinomas and is negatively regulated by the p19ARF tumor suppressor. Genes Dev. 2004;18:830-50.

27. Kim IM, Ackerson T, Ramakrishna S, Tretiakova M, Wang IC, Kalin TV, et al. The Forkhead Box m1 transcription factor stimulates the proliferation of tumor cells during development of lung cancer. Cancer Res. 2006;66:2153-61.

28. Liu M, Dai B, Kang SH, Ban K, Huang FJ, Lang FF, et al. FoxM1B is overexpressed in human glioblastomas and critically regulates the tumorigenicity of glioma cells. Cancer Res. 2006;66:3593-602.

29. Chan DW, Yu SY, Chiu PM, Yao KM, Liu WW, Cheung AN, et al. Over-expression of FOXM1 transcription factor is associated with cervical cancer progression and pathogenesis. J Pathol. 2008;215:245-52.

30. Li Q, Zhang N, Jia Z, Le X, Dai B, Wei D, et al. Critical role and regulation of transcription factor FoxM1 in human gastric cancer angiogenesis and progression. Cancer Res. 2009;69:3501-9.

31. Uddin S, Ahmed M, Hussain A, Abubaker J, Al-Sanea N, AbdulJabbar A, et al. Genome-wide expression analysis of Middle Eastern colorectal cancer reveals FOXM1 as a novel target for cancer therapy. Am J Pathol. 2011:178:537-47

32. Gemenetzidis E, Bose A, Riaz AM, Chaplin T, Young BD, Ali M, et al. FOXM1 upregulation is an early event in human squamous cell carcinoma and it is enhanced by nicotine during malignant transformation. PLoS One. 2009;4, e4849.
33. Teh MT, Hutchison IL, Costea DE, Neppelberg E, Liavaag PG, Purdie K, et al. Exploiting FOXM1-orchestrated molecular network for early squamous cell carcinoma diagnosis and prognosis. Int J Cancer. 2013;132:2095-106.

34. Scheid MP, Woodgett JR. Protein kinases: six degrees of separation? Curr Biol. 2000;10:R191-4.

35. Rahman MA, Amin AR, Shin DM. Chemopreventive potential of natural compounds in head and neck cancer. Nutr Cancer. 2010;62:973-87.

36. Marquette $C$, Nabell L. Chemotherapy-resistant metastatic breast cancer. Curr Treat Options Oncol. 2012;13:263-75.

37. Wang IC, Meliton L, Tretiakova M, Costa RH, Kalinichenko W, Kalin TV. Transgenic expression of the forkhead box M1 transcription factor induces formation of lung tumors. Oncogene. 2008;27:4137-49.

38. Hegde NS, Sanders DA, Rodriguez R, Balasubramanian S. The transcription factor FOXM1 is a cellular target of the natural product thiostrepton. Nat Chem. 2011;3:725-31.

39. Bhat UG, Halasi M, Gartel AL. FoxM1 is a general target for proteasome inhibitors. PLoS One. 2009:4, e6593.

40. Uddin S, Hussain AR, Ahmed M, Siddiqui K, Al-Dayel F, Bavi P, et al. Overexpression of FoxM1 offers a promising therapeutic target in diffuse large B-cell lymphoma. Haematologica. 2012;97:1092-100.

41. Ahmed M, Uddin S, Hussain AR, Alyan A, Jehan Z, Al-Dayel F, et al. FoxM1 and its association with matrix metalloproteinases (MMP) signaling pathway in papillary thyroid carcinoma. J Clin Endocrinol Metab. 2012;97:E1-13.

42. Chou TC, Talalay P. Quantitative analysis of dose-effect relationships: the combined effects of multiple drugs or enzyme inhibitors. Adv Enzyme Regul. 1984;22:27-55.

43. Antonsson B, Montessuit S, Sanchez B, Martinou JC. Bax is present as a high molecular weight oligomer/complex in the mitochondrial membrane of apoptotic cells. J Biol Chem. 2001;276:11615-23.

44. Hussain AR, Ahmed M, Al-Jomah NA, Khan AS, Manogaran P, Sultana M, et al. Curcumin suppresses constitutive activation of nuclear factor-kappa $B$ and requires functional Bax to induce apoptosis in Burkitt's lymphoma cell lines. Mol Cancer Ther. 2008;7:3318-29.

45. Al-Maghrabi J, Buhmeida A, Emam E, Syrjanen K, Sibiany A, Al-Qahtani M, et al. Cyclooxygenase-2 expression as a predictor of outcome in colorectal carcinoma. World J Gastroenterol. 2012;18:1793-9.

46. Li D, Wei P, Peng Z, Huang C, Tang H, Jia Z, et al. The critical role of dysregulated FOXM1-PLAUR signaling in human colon cancer progression and metastasis. Clin Cancer Res. 2013;19:62-72.

47. Seitz C, Hugle M, Cristofanon S, Tchoghandjian A, Fulda S. The dual PI3K mTOR inhibitor NVP-BEZ235 and chloroquine synergize to trigger apoptosis via mitochondrial-lysosomal cross-talk. Int J Cancer. 2012;132(11):2682-93.

48. Peddaboina C, Jupiter D, Fletcher S, Yap JL, Rai A, Tobin R, et al. The downregulation of $\mathrm{MCl}-1$ via USP9X inhibition sensitizes solid tumors to $\mathrm{BCl}-\mathrm{xl}$ inhibition. BMC Cancer. 2012;12:541.

49. Itatsu K, Sasaki M, Yamaguchi J, Ohira S, Ishikawa A, Ikeda H, et al. Cyclooxygenase- 2 is involved in the up-regulation of matrix metalloproteinase-9 in cholangiocarcinoma induced by tumor necrosis factor-alpha. Am J Pathol. 2009;174:829-41.

50. Bu X, Zhao C, Dai X. Involvement of COX-2/PGE (2) Pathway in the Upregulation of MMP-9 Expression in Pancreatic Cancer. Gastroenterol Res Pract. 2011;2011:214269.

51. Bartholomeusz C, Gonzalez-Angulo AM. Targeting the PI3K signaling pathway in cancer therapy. Expert Opin Ther Targets. 2012;16:121-30.

52. Yao M, Lam EC, Kelly CR, Zhou W, Wolfe MM. Cyclooxygenase-2 selective inhibition with NS-398 suppresses proliferation and invasiveness and delays liver metastasis in colorectal cancer. Br J Cancer. 2004:90:712-9.

53. Bavi P, Prabhakaran SE, Abubaker J, Qadri Z, George T, Al-Sanea N, et al. Prognostic significance of TRAIL death receptors in Middle Eastern colorectal carcinomas and their correlation to oncogenic KRAS alterations. Mol Cancer. 2010;9:203

54. Bavi P, Jehan Z, Atizado V, Al-Dossari H, Al-Dayel F, Tulbah A, et al. Prevalence of fragile histidine triad expression in tumors from saudi arabia: a tissue microarray analysis. Cancer Epidemiol Biomarkers Prev. 2006;15:1708-18.

55. Camp RL, Dolled-Filhart M, Rimm DL. X-tile: a new bio-informatics tool for biomarker assessment and outcome-based cut-point optimization. Clin Cancer Res. 2004;10:7252-9. 
56. Kwok JM, Myatt SS, Marson CM, Coombes RC, Constantinidou D, Lam EW. Thiostrepton selectively targets breast cancer cells through inhibition of forkhead box M1 expression. Mol Cancer Ther. 2008;7:2022-32.

57. Hussain AR, Ahmed SO, Ahmed M, Khan OS, Al Abdulmohsen S, Platanias LC, et al. Cross-talk between NFkB and the PI3-kinase/AKT pathway can be targeted in primary effusion lymphoma (PEL) cell lines for efficient apoptosis. PLoS One. 2012;7, e39945.

58. Hussain AR, Al-Rasheed M, Manogaran PS, Al-Hussein KA, Platanias LC, Al Kuraya K, et al. Curcumin induces apoptosis via inhibition of PI3'-kinase/AKT pathway in acute T cell leukemias. Apoptosis. 2006;11:245-54.

\section{Submit your next manuscript to BioMed Central} and take full advantage of:

- Convenient online submission

- Thorough peer review

- No space constraints or color figure charges

- Immediate publication on acceptance

- Inclusion in PubMed, CAS, Scopus and Google Scholar

- Research which is freely available for redistribution 